\title{
Mental practice: a simple tool to enhance team-based trauma resuscitation
}

Gianni R. Lorello, MD, MSc*; Christopher M. Hicks, MD, MEd ${ }^{\dagger}$; Sana-Ara Ahmed, MD*; Zoe Unger, MD, MEd*; Deven Chandra, MD, MEd*; Megan A. Hayter, MD, MEd ${ }^{\ddagger}$

\section{ABSTRACT}

Introduction: Effective trauma resuscitation requires the coordinated efforts of an interdisciplinary team. Mental practice (MP) is defined as the mental rehearsal of activity in the absence of gross muscular movements and has been demonstrated to enhance acquiring technical and procedural skills. The role of MP to promote nontechnical, team-based skills for trauma has yet to be investigated.

Methods: We randomized anaesthesiology, emergency medicine, and surgery residents to two-member teams randomly assigned to either an MP or control group. The MP group engaged in 20 minutes of MP, and the control group received 20 minutes of Advanced Trauma Life Support (ATLS) training. All teams then participated in a high-fidelity simulated adult trauma resuscitation and received debriefing on communication, leadership, and teamwork. Two blinded raters independently scored video recordings of the simulated resuscitations using the Mayo High Performance Teamwork Scale (MHPTS), a validated team-based behavioural rating scale. The Mann-Whitney U-test was used to assess for between-group differences.

Results: Seventy-eight residents provided informed written consent and were recruited. The MP group outperformed the control group with significant effect on teamwork behaviour as assessed using the MHPTS: $r=0.67, p<0.01$.

Conclusions: MP leads to improvement in team-based skills compared to traditional simulation-based trauma instruction. We feel that MP may be a useful and inexpensive tool for improving nontechnical skills instruction effectiveness for team-based trauma care.

\section{RÉSUMÉ}

Introduction: La réanimation efficace des traumatisés exige la coordination des efforts d'une équipe interdisciplinaire. La visualisation mentale (VM) se définit comme la répétition mentale d'une action, sans mouvement musculaire visible, et il a été démontré que ce type d'exercice mental améliorait
I'acquisition de compétences techniques et opératoires. Toutefois, le rôle de la VM visant à faciliter l'acquisition de compétences non techniques, réalisées en équipe, dans la prestation des soins aux traumatisés reste à étudier.

Méthode: Après répartition aléatoire, des résidents en anesthésiologie, en médecine d'urgence et en chirurgie ont été divisés en équipes de deux membres, elles-mêmes dirigées au hasard vers le groupe de VM ou vers le groupe témoin. Le groupe de VM s'est concentré sur une VM de 20 minutes, tandis que le groupe témoin a reçu une formation de 20 minutes sur les principes des soins avancés de réanimation traumatologique (Advanced Trauma Life Support $^{(}$[ATLS]). Par la suite, toutes les équipes ont participé à une simulation réaliste de réanimation chez des adultes traumatisés, après quoi elles ont reçu un compte rendu sur la communication, le pouvoir d'influence et le travail d'équipe. Deux examinateurs tenus dans l'ignorance des faits ont évalué, chacun de leur côté, les enregistrements vidéo des réanimations simulées, et ce, à l'aide de la Mayo High Performance Teamwork Scale (MHPTS), une échelle validée d'évaluation du comportement en équipe. Le test $U$ de MannWhitney a servi à évaluer les écarts entre groupes.

Résultats: Soixante-dix-huit résidents ont donné, par écrit, leur consentement éclairé, puis ont été retenus pour l'étude. Le groupe de VM a obtenu des résultats significativement meilleurs que le groupe témoin en ce qui concerne le comportement en équipe, d'après la Mayo Team Scale (MHPTS: $r=0,67 ; p<0,01$ ).

Conclusions: La VM a permis d'améliorer les compétences liées au travail d'équipe comparativement à la formation classique, fondée sur la simulation, sur les soins à donner aux traumatisés. Les auteurs sont d'avis que la VM pourrait s'avérer un outil utile et économique, qui permettrait d'améliorer l'efficacité de la formation sur les compétences non techniques, dans la prestation des soins en équipe, en traumatologie.

Keywords: nontechnical skills, simulation, trauma

From the Departments of *Anaesthesiology and the Li Ka Shing Knowledge Institute; †Emergency Medicine and the Li Ka Shing Knowledge Institute, St. Michael's Hospital, University of Toronto, Toronto, ON; and ¥Department of Anaesthesia, Rockyview General Hospital, University of Calgary, Calgary, AB.

Correspondence to: Dr. Christopher Hicks, Department of Emergency Medicine, St. Michael's Hospital, 30 Bond Street, 1-008e Shuter Wing, Toronto, ON M5B 1W8; Email: hicksc@smh.ca

This article was originally published as Original Research. It was intended to be Education. 


\section{INTRODUCTION}

Trauma resuscitation is a dynamic, high-stakes team activity. Successful management of complex trauma patients depends not only on diagnostic and technical ability, but also on team-based skills, such as effective leadership and communication, efficient resource utilization, role clarity, and situation awareness. High functioning multimember teams can mitigate the effects of extreme time pressures, decision density, and ambient noise and distractions by distributing tasks, assigning roles, and cross-monitoring performance.

The work environment for health care professionals is increasingly becoming team-based. The World Health Organization has endorsed the importance of training health care teams as a strategy to improve team cohesion, promote patient safety, and reduce medical error. ${ }^{1}$ Simulation-based team training is an effective method for promoting the acquisition of key nontechnical team behaviours, but it is expensive, resource intensive, and time-consuming. A need exists to develop instructional methods that improve both the efficiency and effectiveness of simulation-based team training.

Mental practice (MP) is defined as the "cognitive rehearsal of a skill in the absence of an overt physical movement" ${ }^{2}$ and specifically refers to a training technique in which the procedure and the specific steps necessary to complete it are mentally imagined and covertly rehearsed before performance. MP encompasses elements of imagery practice commonly used by elite athletes and musicians, and is considered to be a productive and cost-effective means for learning and enhancing performance in other domains., ${ }^{2,3}$

MP has been shown to improve technical skill performance in surgical skills training, ${ }^{4-8}$ and a recent meta-analysis found that, although MP influences performance on both cognitive and physical tasks, it has a greater influence on tasks requiring a larger mental workload (i.e., high-stakes situations) than tasks requiring strength and coordination. ${ }^{2}$ The effect of MP on team performance and nontechnical skills is currently not known.

The purpose of this study was to examine the effect of 20 minutes of structured MP on team performance in a simulated trauma resuscitation. Our primary outcome was nontechnical skills acquisition and execution as assessed by two blinded reviewers using a previouslyvalidated behavioural rating scale, the Mayo High Performance Teamwork Scale (MHPTS).
METHODS

\section{Study design and setting}

This was a prospective, single-blinded, randomized, simulation-based study. All participants received a standardized orientation to the high-fidelity mannequin (SimMan Laerdal, Toronto, ON) and the simulated trauma environment (Allan Waters Family Simulation Centre, St. Michael's Hospital, Toronto, ON).

\section{Selection of participants}

After receiving research ethics board approval (St. Michael's Hospital Research Ethics Board, Toronto, $\mathrm{ON}$ ), we invited 78 postgraduate trainees (residents in years 1 through 5) from the Departments of Anesthesiology, Emergency Medicine, and General Surgery at the University of Toronto to participate. Prior to the study launch, a research assistant blinded to the study aims formed teams by randomizing trainees into pairs of two, one from anesthesiology (trauma team member) and the other from either emergency medicine or general surgery (trauma team leader). This same blinded research assistant subsequently randomized the two-member teams to either the control group or MP group by random number assignment on the day of training. Written informed consent was obtained for all participants prior to training, including permission to be videotaped.

\section{Interventions}

Participants in the MP group participated in 20 minutes of quiet mental rehearsal of a descriptive script based on key Advanced Trauma Life Support (ATLS, American College of Surgeons, Chicago, IL, USA) principles and teamwork skills (Appendixes 1A and 1B). Participants were instructed to visualize a trauma scenario and how they would behave and function in a team while reviewing the script. The participants were allowed to discuss the script with their fellow team member. The 20-minute MP allocation time was chosen based on a previously-published meta-analysis examining the optimal duration for MP for both cognitive and physical tasks. ${ }^{2}$ Participants in the control group received 20 minutes of face-to-face teaching on the ATLS algorithm and nontechnical elements of team-based trauma care. Following either the 20 minutes of ATLS 
training and MP or 20 minutes of ATLS teaching alone, all participants completed a modified mental imagery questionnaire (mMIQ) designed to evaluate an individual's mental imagery aptitude (Appendix 2).

Immediately following training, all teams participated in a high-fidelity adult trauma simulation, which was videotaped for a future blinded review. Teams received debriefing based on their resuscitation performance, which was excluded from the videotaping and review.

\section{Mental practice script}

The MP scripts were text documents that described the procedural steps of ATLS trauma principles and protocols, tailored to either the team leader or team member role. They contained key visual, cognitive, and kinesthetic cues believed to be important for cohesive team performance in a symbolic trauma resuscitation. The authors developed the MP scripts using a combination of established ATLS protocols and focused interviews with anesthesiologists and trauma team leaders to determine the key cues that promote effective teamwork skills.

\section{Simulation scenario}

Two of the study authors (CMH and $\mathrm{MH})$, both experts in trauma, anesthesiology, and medical education, reviewed the scenario for content validity. The scenario was pilot tested and revised using volunteer teams who did not participate in the evaluative component. All study scenarios were completed in a simulated trauma bay using a high-fidelity mannequin with standard noninvasive monitors applied (blood pressure cuff, electrocardiogram leads II and V5, pulse oximeter). The scenario involved a pregnant trauma patient at 28 weeks' gestational age involved in a high-speed motor vehicle crash, with an altered level of consciousness and obstructive shock from a left tension pneumothorax (Appendix 3). The scenario was terminated as soon as the trauma team initiated needle decompression of the left tension pneumothorax or when a total of 15 minutes had elapsed without this procedure taking place. Two confederates played the scripted roles of an emergency department trauma nurse and a distressed family member, and they were instructed to interact with team members in a standardized fashion. The confederates were permitted to prompt participants when key data, such as shock or persistent hypoxemia, were being overlooked. The two confederates remained blinded to the study interventions. All scenarios were video recorded for subsequent blinded video analysis by two independent reviewers, and a graphical display of the vital signs was superimposed on the video footage.

\section{Outcome measures}

The primary end point was the effect of MP on trauma teamwork performance as measured by the Mayo High Performance Teamwork Scale (MHPTS). The MHPTS is a previously-validated behavioural rating scale for high performance teamwork skills in a simulated environment. ${ }^{9}$ It is composed of eight positive teamwork questions and eight negative teamwork questions (each on a 3-point Likert scale) derived from crisis resource management (CRM) principles of communication, leadership, situation awareness, and problem solving. The inter-rater reliability for the MHPTS has been found to be moderate, and the MHPTS performs similarly to the Ottawa Global Rating Scale (GRS) when novice-novice raters, expertexpert raters, and novice-expert raters are compared. ${ }^{10}$

Our secondary end point was the participant's score on the mMIQ. The mMIQ was applied to control for individual variation in mental imagery aptitude. ${ }^{5}$ The mMIQ is based on a 7-point Likert scale composed of eight questions and serves as a manipulation check to assess the vividness of an individual's imagery process prior to participating in an activity. ${ }^{5}$

\section{Statistical analysis}

The video recorded scenarios were uploaded into StudioCode (StudioCode Business Group, Canada Headquarters, Toronto, ON), a proprietary video analysis software program. Two physicians with expertise in teamwork and trauma resuscitation were recruited to analyse the videotaped data. To calibrate the data collection process, the raters viewed and scored two study videos using the MHPTS and were permitted to discuss their results, while remaining blinded to group allocation. The raters then independently scored the remaining scenarios, and the data were abstracted from StudioCode for statistical analysis.

The unit of analysis was each individual team. Sample size was calculated a priori according to a power analysis calculation based on previous work with MP (two-tailed alpha of 0.05 , power of 0.80 , Cohen's d effect size of 0.80 ), resulting in a total sample size requirement of 34 groups. ${ }^{11}$ Nonparametric analysis sample size calculation 
adjustment was performed using a Pitman asymptotic relative efficiency (ARE), which compares the relative efficiency of two competing tests (i.e., the MannWhitney $\mathrm{U}$ test compared to the independent student t-test), thereby resulting in the ratio of sample sizes required for equal power using the two tests. The lower limit of the ARE (i.e., the more conservative relative efficiency) for this power analysis calculation is 0.86 , which resulted in a total sample size requirement of 39 (i.e., 34/0.86 = 39) groups.

The Mann-Whitney $U$ test was used to assess for between-group differences in the MHPTS (our primary outcome) and the mMIQ (our secondary outcome). Participant demographic data, such as postgraduate training level, gender, and number of previous simulation experiences, were analysed using the Fisher's exact test, the Mann-Whitney-U test, and the independent student t-tests as appropriate. For all data analysed by the Mann-Whitney U test, a standardized effect size (r) was calculated where the $\mathrm{z}$-score was converted into the effect size (ES) estimate, $r$, where $r=0.1$ indicates a small $\mathrm{ES}$, $\mathrm{r}=0.3$ indicates a moderate $\mathrm{ES}$, and $\mathrm{r}=0.5$ indicates a large ES. ${ }^{12}$

Inter-rater reliability was calculated according to a two-way mixed, single measures intra-class correlation (ICC), an equivalent approach to the weighted kappa. ${ }^{13,14}$

All statistical analyses were performed using SPSS (IBM Version 21).

\section{RESULTS}

\section{Characteristics of study participants}

Seventy-eight residents were recruited and completed the study: 19 teams in the control group and 20 teams in the MP group. There were no statistically significant between-group differences in demographics (Table 1). The median number of postgraduate years (PGY) of training for participants in the control group was three, compared to two in the MP group $(p=0.06)$.

\section{Main results}

The ICC for the MHPTS was 0.6. The MP group significantly outperformed the control group, having a strong effect favouring MP on teamwork behaviour on the MHPTS $(\mathrm{r}=0.67, p<0.01)$. The median score for the MP group was 21.5 (inter-quartile range [IQR] 21-22.5), and the median score for the control group was 19 (IQR: 18-20) out of a total potential score of 24 (Figure 1).

The mMIQ total scores for the control group and the MP group (Figure 2) were also significantly different $(p=0.03)$.

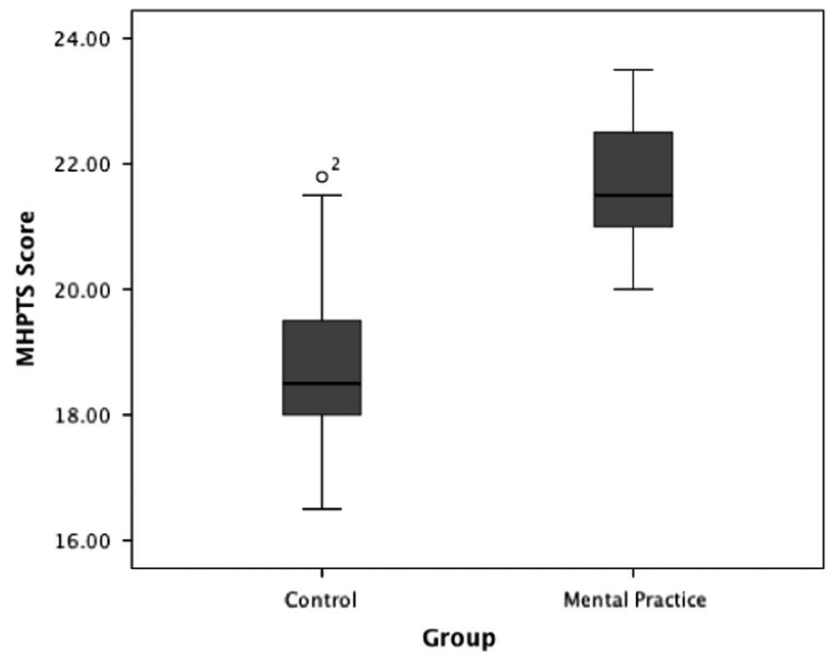

Figure 1. Box plot depicting the Mayo High Performance Teamwork Scale (MHPTS) results (minimum score $=0$; maximum score $=24$ ) for the control and mental practice groups.

The central bar represents the median, the box represents the interquartile range, and the bars represent the data range.

Table 1. Baseline participant demographics.

\begin{tabular}{lcc|}
\hline & Control Group $(n=38)$ & Mental Practice Group $(n=40)$ \\
\hline Age (years), Median (SD) & $29.1(3.2)$ & $29.4(3.6)$ \\
\# Male (\%) & $24(63.2)$ & $22(55)$ \\
PGY Level, Median (IQR) & $3(2-3)$ & 87.5 \\
$\%$ Yes Previous Exposure to Simulation & 97.4 & $6.3(6.4)$ \\
Previous Mean \# Hours in Simulation (SD) & $7.0(5.1)$ & \\
SD = Standard deviation & & \\
IQR = Interquartile range & & \\
$\mathrm{Cl}=$ Confidence Interval & & \\
\hline
\end{tabular}




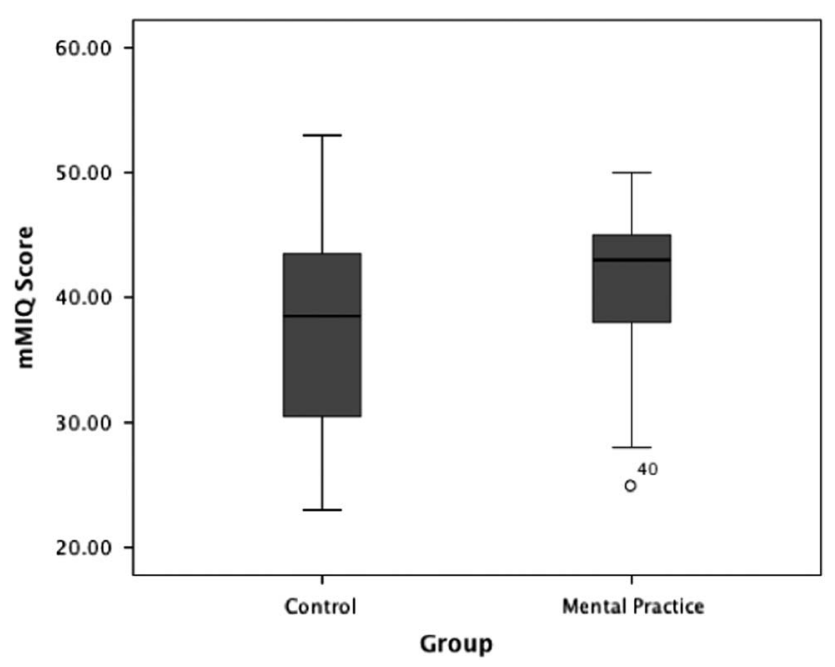

Figure 2. Box plot demonstrating the modified Mental Imagery Questionnaire ( $\mathrm{mMIO}$ ) results (minimum score $=8$; maximum score $=56$ ) for the control and mental practice groups.

The central bar represents the median, the box represents the interquartile range, and the bars represent the data range.

\section{DISCUSSION}

In a variety of high-stakes fields including athletics, music, and more recently surgery, MP has been shown to be a powerful tool for enhancing task performance. ${ }^{4-8,15-17}$ The current evidence in surgical disciplines suggests that MP is a safe and cost-effective method for training novice surgeons in technical procedures. ${ }^{8,16,17}$ A randomized controlled trial by Arora et al. found MP to be beneficial for enhancing the imagery and performance of novice surgeons during a simulated laparoscopic cholecystectomy. ${ }^{5,8}$ Recently, Hayter et al. found that warm-up using MP did not improve anesthesia CRM skills in novice anesthesia trainees during a simulated cardiac arrest. ${ }^{11}$ This study found no significant difference in performance of CRM skills as assessed by the Ottawa GRS, or on the secondary outcomes of time to chest compressions, time to epinephrine administration, and time to blood administration. One possible explanation for this is that novice trainees may not have the foundational skills necessary to perform as team leader during a complex resuscitation. Moreover, the MP script used in Hayter's study likely did not contain sufficient verbal cues to stimulate the visual and kinesthetic rehearsal believed to be an important aspect of skill acquisition. ${ }^{11}$

Our results suggest that 20 minutes of MP script rehearsal is associated with improved team behaviour during a simulated trauma resuscitation. We found a significant difference in team performance favouring MP as assessed by the MHPTS. To our knowledge, ours is the first study to demonstrate a positive effect of MP on team-based behaviours in the trauma environment. Furthermore, although our control group was, by chance, composed of more experienced trainees, we still observed a significant effect of the intervention favouring the less experienced MP group.

As situation complexity increases and stress accumulates, mental models of team-based processes break down, leading to a lack of global perspective and team cohesiveness. ${ }^{18,19}$ During a stressful event, loss of situation awareness begins first with ignoring peripheral cues, then task-relevant cues, errors of fixation, and premature diagnostic closure. ${ }^{18,19}$ Complex clinical events that require multitasking or contain multiple data sources are particularly vulnerable to cognitive tunneling (the exclusion of potentially relevant diagnostic information due to fixation on a particular feature of the clinical scenario). ${ }^{18,19}$ Cognitive tunneling may be mitigated, in part, by a team-based approach, where any team member can prompt the team leader to redirect his or her attention to potentially relevant diagnostic cues. MP performed as a team may support developing effective shared mental models for team-based and task-based processes. We hypothesize that these shared mental modes may be one mechanism by which MP lead to improved team performance in the intervention group.

MP is grounded in dual-coding theory, which posits that, although cognition comprises both verbal and nonverbal activity, separate processing mechanisms exist for both imagery and linguistic activity. ${ }^{4}$ Accordingly, memory and recall are enhanced when they are encoded from both verbal and nonverbal cognition. ${ }^{11}$ In our study, the MP group was found to have significantly higher scores on the mMIQ, suggesting that they were more actively engaged in thinking about key trauma principles. This may have helped team leaders clarify team and taskbased processes in advance, thus decreasing cognitive load and increasing the amount of available working memory for complex tasks, such as problem solving and maintaining situational awareness. MP may also have acted as a visual repetitive action for technical skills, giving participants clear, process-oriented goals.

\section{LIMITATIONS}

Our study has several important limitations. The original trauma resuscitation scenario involved a traumatic cardiac 
arrest and cardiopulmonary resuscitation (CPR). A trauma arrest scenario was felt to add a level of technical complexity that could confound team-based goals. As a result, the scenario was adjusted to eliminate the need for CPR in favour of highly unstable vital signs that could be fully mitigated by needle decompression and chest tube insertion. This early change in the resuscitation content may have precluded comparison between the groups who received or did not receive the modified scenario script. However, only two scenarios included a full cardiac arrest before the resuscitation script was altered, and a reanalysis of our data, excluding the two modified scenarios, yielded similar overall results without changing the direction, magnitude, or statistical significant effect of MP on team behaviours as assessed by the MHPTS.

The modest ICC we found of 0.6 may have arisen due to poor psychometric properties of the MHPTS or inadequate rater training. ${ }^{10}$ Still, this ICC remains well above the ICC observed by Iwashita et al. when determining the reliability and validity of the MHPTS compared to the Ottawa GRS in a simulated crisis teamwork course. ${ }^{10}$

Some participants may have a better capacity to partake in MP than others, and we did not control for MP capacity (as assessed by the mMIQ) during the randomization process. Future research on MP could stratify group allocation based on the capacity to engage in MP or recruit a sufficient number of participants to perform subgroup analyses based on mMIQ scores. Although we infer that participants engaging in MP were focusing on nontechnical skills during their rehearsal period, we cannot know this with certainty. Exploratory research using qualitative methods may permit a better understanding of exactly what was being "rehearsed" during the MP exercise: CRM principles (as the script dictated) or other abilities, such as MP of anticipated technical skills. In addition, retention tests could be performed to help identify the ideal interval for refresher training.

Finally, we did not train nurses, respiratory therapists, or other inter-professional team members, which may limit the generalizability of our results. Future efforts should focus on MP in a truly inter-professional context to enhance its relevance to emergency and trauma teams.

\section{CONCLUSION}

MP leads to improvement in team-based skills compared to traditional simulation-based trauma instruction.
We feel that MP may be a useful and inexpensive tool for improving nontechnical skills instruction effectiveness for team-based trauma care. Training with MP may serve the dual purpose of increasing the impact of simulation-based instruction for trauma teams and providing team members with a useful cognitive tool to formalize the "elevator rehearsal" prior to engaging in complex team-based patient care. Future research should focus on the feasibility of inter-professional MP instruction and examine both skill retention and impact on patient-oriented outcomes.

Acknowledgements: The authors would like to thank Roger Chow and Tetyana Postonogova for their help with the simulation scenarios. We would also like to thank Simon Reynolds from StudioCode for his time in demonstrating the software and for the temporary licence during this study.

Competing interests: None declared. Authors' funding sources are attributed to Innovation Fund 2010, St. Michael's Hospital, Toronto, ON.

\section{SUPPPLEMENTARY MATERIAL}

To view supplementary material for this article, please visit http://dx.doi.org/10.1017/cem.2015.4

\section{REFERENCES}

1. Yan J, Gilbert JHV, Hoffman SJ. WHO Study Group on Interprofessional Education and Collaborative Practice. 7 Interprof Care 2007;21(6):587-9.

2. Driskell JE, Copper C, Moran A. Does mental practice enhance performance? $\mathcal{F}$ Appl Psychol 1994;79:481-92.

3. Weinberg R. Does imagery work? Effects on performance and mental skills. 7 Imagery Res Sport Phys Activ 2008; 3(1):1-21.

4. Sanders CW, Sadoski M, Bramson R, et al. Comparing the effects of physical practice and mental imagery rehearsal on learning basic surgical skills by medical students. Am 7 Obstet Gynecol 2004;191(5):1811-4.

5. Arora S, Aggarwal R, Sevdalis N, et al. Development and validation of mental practice as a training strategy for laparoscopic surgery. Surg Endosc 2010;24(1):179-87.

6. Komesu Y, Urwitz-Lane R, Ozel B, et al. Does mental imagery prior to cystoscopy make a difference? A randomized controlled trial. Am 7 Obstet Gynecol 2009;201(2): 218.e1-9.

7. Immenroth M, Bürger T, Brenner J, et al. Mental training in surgical education: a randomized controlled trial. Ann Surg 2007;245(3):385-91.

8. Arora S, Aggarwal R, Sirimanna P, et al. Mental practice enhances surgical technical skills: a randomized controlled study. Ann Surg 2011;253(2):265-70.

9. Malec JF, Torsher LC, Dunn WF, et al. The mayo high performance teamwork scale: reliability and validity for 
evaluating key crew resource management skills. Simul Healthc: $\mathcal{F}$ Soc Simul Healthc 2007;2(1):4-10.

10. Iwashita H, Emlet L, Berg B, et al. Validation of the Mayo High Performance Teamwork Scale and Ottawa Crisis Resource Management Global Rating Scale for Evaluation of Crisis Team Training. WISER, 20 Years of Simulations Improving Healthcare Society for Simulation in Healthcare 2014: abstract.

11. Hayter MA, Bould MD, Afsari M, et al. Does warm-up using mental practice improve crisis resource management performance? A simulation study. Br 7 Anaesth 2013; 110(2):299-304.

12. Cohen J. A power primer. Psychol Bull 1992;112(1):155-9.

13. Cohen J. Weighted kappa: nominal scale agreement with provision for scaled disagreement or partial credit. Psychol Bull 1968;70(4):213-20.
14. Fleiss JL. The equivalence of the weighted kappa and the intraclass correlation coefficient as measures of reliability. Educ Psychol Meas 1973;33(3):613-9.

15. Calatayud D, Arora S, Aggarwal R, et al. Warm-up in a virtual reality environment improves performance in the operating room. Ann Surg 2010;251(6):1181-5.

16. Driskell J. Group decision making under stress. $7 \mathrm{Appl}$ Psychol 1991;76:473-8.

17. Driskell J, Salas E, Johnston J. Does stress lead to a loss of team perspective? Group Dyn-Theor Res 1999;3(4):291-302.

18. Mathieu JE, Heffner TS, Goodwin GF, et al. The influence of shared mental models on team process and performance. 7 Appl Psychol 2000;85(2):273-83.

19. Landis JR, Koch GG. The measurement of observer agreement for categorical data. Biometrics 1977;33(1): $159-74$. 